\title{
Radiation Cholecystitis: A Diagnosis to Be Considered?
}

\author{
Kaushik M*, Breeden J and Mittal V \\ Department of General Surgery, Ascension Providence Hospital, Michigan State \\ University, USA
}

*Corresponding author: Manu Kaushik MD, Department of General Surgery, Ascension

\section{Editorial \\ Volume 3 Issue 1}

Received Date: August 19, 2019

Published Date: August 26, 2019

Providence Hospital, Michigan State University, Southfield MI, USA, Email: manukaushik88@gmail.com

\section{Editorial}

Radiation cholecystitis is an uncommon biliary complication after yttrium -90 radio-embolization. The incidence of radiation cholecystitis is almost $1 \%$ after radio-embolization. The spectrum of this complication can range from biliary dyskinesia to acute cholecystitis. This complication is a result of non-targeted radioembolization of microspheres in the capillary network supplying the gall bladder wall via the cystic artery [1]. Most studies and reviews state that this complication occurs because of penetration of regional hepatic tissue, however, this can be refuted given that the mean penetration of emitted Beta particles is $2.5 \mathrm{~mm}$ with a maximum penetration of $10 \mathrm{~mm}$ [1]. A careful consideration of the hepatic anatomy and the positioning of the angiographic catheter during the mapping and dose delivery can significantly prevent the risk of radiation induced biliary complications.

Radio-embolization with yttrium microspheres has become an established therapeutic modality in management for primary and secondary hepatic malignancies [2]. Some centers have attempted to use technicium labeled micro-aggregated albumin infusion into the intended $90 \mathrm{Y}$ infusion artery, and nuclear imaging can estimate the area of eventual distribution. Despite all precautionary measures, radiation cholecystitis remains to be a potential complication with a high morbidity and mortality.

The biggest challenge faced by a General surgeon is diagnosing this rare complication and managing it appropriately. Most of these patients present to the emergency department with clinical signs and symptoms of acute cholecystitis and are often operated upon without consideration of radiation cholecystitis. In a series by Atassi et al, reported 6 patients with gallbladder enhancement and 3 patients with asymptomatic gall bladder wall disruption, of whom 2 patients ended up with a cholecystectomy [2]. Cholecystectomy is rarely needed for these patients and in most cases, conservative management will resolve symptoms and patients recover without any sequelae or further attacks of cholecystitis. A small number of case reports have shown the benefits of conservative management, avoiding unnecessary surgical intervention. Hence, diagnosis of radiation-induced cholecystitis relies largely on the clinical profile of the patient. In most cases, the imaging findings may not correlate with the clinical severity or need of an operation. A majority of these patients will have significant imaging findings even on follow-up but remain asymptomatic.

The treatment of radiation cholecystitis should focus upon supportive measures like hydration, antibiotics and analgesics. If patients fail to progress or have symptoms for more than 2 weeks, Surgical intervention can be considered as the half-life of $90 \mathrm{Y}$ is 64 hours [3]. Prognosis or post discharge symptomology depends largely upon the severity of the initial symptoms and response to conservative management. Most reports show that symptomatic management is adequate for managing these patients and only a minority of this patient population required a cholecystectomy. For the operating surgeon, it is important to consider factors like bleeding complications in patients with metastatic hepatic disease with an element of cirrhosis.

In conclusion, from our experience and an extensive review of literature, we feel it is safe to state that radiation cholecystitis should be considered when a 


\section{International Journal of Surgery \& Surgical Techniques}

patient presents with abdominal pain post radio embolization with evidence of cholecystitis vs biliary dyskinesia on imaging. These patients require a multidisciplinary approach involving not just the surgical services but also radiologists to evaluate the imaging closely with a high degree of suspicion and a thorough clinical evaluation.

\section{References}

1. Parakh S, Gananadha S, Allen RYip D (2016) Cholecystitis after yittrium-90 resin microsphere radioembolization treatment: Clinical and pathologic findings. Asian J Surg 39(3): 144-148.

2. Atassi B, Bangash A K, Lewandowski R J, Ibrahim S, Kulik L et al. (2008) Biliary sequelae following radio embolization with Yttrium-90 microspheres. J Vasc Interv Radiol 19(5): 691-697.

3. Hickey R, Lewandowski R, Hepatic (2011) Radio embolization Complicated by Radiation Cholecystitis: Semin Intervent Radiol 28(2): 230-233. 